\title{
A VARIATIONAL PROBLEM IN REACTOR THEORY
}

J. ERNEST WILKINS, JR.

1. Introduction. Let $C$ be a bounded measurable set of points in three-dimensional Euclidean space. ${ }^{1}$ Let $H(x, y)$ be symmetric in its arguments, in $L_{2}(C, C)$, and not almost everywhere zero. Let $u(x)$ be a bounded, measurable, non-negative function on $C$ which is not almost everywhere zero. If in addition there exists a non-negative function $v(x)$ in $L_{2}(C)$ which is not almost everywhere zero such that

$$
v(x)=\int_{C} H(x, y) u(y) v(y) d y,
$$

then we shall say that $u(x)$ is in the class $U$. The problem ${ }^{2}$ is to find a function $u_{0}(x)$ in $U$ which minimizes the integral

$$
I[u]=\int_{C} u(x) d x
$$

on $U$.

If $u_{0}(x)$ is in $U$, then it is known [2] that there exists a finite or denumerable sequence $\lambda_{n}$ of real characteristic values and a corresponding sequence $v_{n}(x)$ of characteristic functions in $L_{2}(C)$ such that

$$
\begin{aligned}
\lambda_{n} \int_{C} H(x, y) u_{0}(y) v_{n}(y) d y & =v_{n}(x), \\
\int_{C} u_{0}(x) v_{m}(x) v_{n}(x) d x & =\delta_{m n} .
\end{aligned}
$$

The theorem we wish to prove may be stated as follows:

Theorem. If $u_{0}(x)$ is in $U$, if $\lambda_{n} \leqq \lambda_{1}<\lambda_{0}=1(n=2,3, \cdots)$, and if the characteristic solution $v_{0}(x)$ is constant, then

$$
\int_{C} u(x) d x \geqq \int_{C} u_{0}(x) d x
$$

whenever $u(x)$ is in $U$ and $u(x) \geqq \lambda_{1} u_{0}(x)$ with equality holding if and

Received by the editors November 16, 1953.

1 The restrictions to three dimensions and to Euclidean space are of course unnecessary.

2 The physical background of this problem can be found in a paper by Goertzel [1], which also gives examples. 
only if

$$
\int_{C} H(x, y)\left[u(y)-u_{0}(y)\right] d y=0 .
$$

2. Proof of the theorem. Let $u(x)$ be in $U$ and suppose that $v(x)$ is a non-negative function in $L_{2}(C)$ which is not almost everywhere zero and for which equation (1) holds. By hypothesis

$$
\int_{C} H(x, y) u_{0}(y) d y=1 .
$$

If we multiply equation (1) by $u_{0}(y)$ and integrate, we find if we make use of the symmetry of $H$ and equation (3) that

$$
\int_{C}\left[u(x)-u_{0}(x)\right] v(x) d x=0 .
$$

Define the sequence $k_{n}^{\prime}$ so that

$$
k_{n}^{\prime}=\int_{C} u(x) v(x) v_{n}(x) d x .
$$

It is clear that $k_{0}^{\prime} \geqq 0$. In fact, $k_{0}^{\prime}>0$, since otherwise $u(x) v(x)$ would vanish almost everywhere and so would $v(x)$ by virtue of equation (1).

It follows from equation (4) that

$$
\begin{aligned}
\int_{C}\left[u(x)-u_{0}(x)\right] d x= & -\int_{C} \frac{\left[u(x)-u_{0}(x)\right] v(x)\left[v(x)-k_{0}^{\prime} v_{0}\right]}{\left(k_{0}^{\prime} v_{0}\right)^{2}} d x \\
& +\left(\lambda_{1}-1\right) \int_{C} u_{0}(x)\left[\frac{v(x)-k_{0}^{\prime} v_{0}}{k_{0}^{\prime} v_{0}}\right]^{2} d x \\
& +\int_{C}\left[u(x)-\lambda_{1} u_{0}(x)\right]\left[\frac{v(x)-k_{0}^{\prime} v_{0}}{k_{0}^{\prime} v_{0}}\right]^{2} d x
\end{aligned}
$$

It is known [2] that

$$
v(x)=\text { I.i.m. } \sum_{n \geqq 0} \frac{k_{n}^{\prime} v_{n}(x)}{\lambda_{n}},
$$

and consequently the first two terms on the right-hand side of equation (5) are

$$
\sum_{n \geqq 1} \frac{\left(1-\lambda_{n}\right) k_{n}^{2}}{\lambda_{n}^{2}}, \quad\left(\lambda_{1}-1\right) \sum_{n \geqq 1} \frac{k_{n}^{2}}{\lambda_{n}^{2}}
$$


in which $k_{n}=k_{n}^{\prime} / k_{0}^{\prime} v_{0}$. Hence

$$
\begin{aligned}
\int_{C}\left[u(x)-u_{0}(x)\right] d x & =\sum_{n \geqq 1} \frac{\left(\lambda_{1}-\lambda_{n}\right) k_{n}^{2}}{\lambda_{n}^{2}} \\
& +\int_{C}\left[u(x)-\lambda_{1} u_{0}(x)\right]\left[\frac{v(x)-k_{0}^{\prime} v_{0}}{k_{0}^{\prime} v_{0}}\right]^{2} d x \geqq 0,
\end{aligned}
$$

since $\lambda_{n} \leqq \lambda_{1}, u(x) \geqq \lambda_{1} u_{0}(x)$.

The inequality (6) is strict unless there is a positive integer $N$ such that $\lambda_{1}=\lambda_{2}=\cdots=\lambda_{N}, k_{N+1}=k_{N+2}=\cdots=0$, and a measurable subset $C_{1}$ of $C$ such that $u(x)=\lambda_{1} u_{0}(x)$ almost everywhere on $C_{1}$ while $v(x)=k_{0}^{\prime}, v_{0}$ almost everywhere on $C-C_{1}$. In this case, let $w(x)$ be defined so that $v(x)=k_{0}^{\prime} v_{0}+w(x)$. Then $w(x)=0$ almost everywhere on $C-C_{1}$,

$$
\begin{gathered}
\lambda_{1} w(x)=\sum_{n=1}^{N} k_{n}^{\prime} v_{n}(x), \\
\lambda_{1} \int_{C} H(x, y) u_{0}(y) w(y) d y=w(x), \\
u(x) w(x)=\lambda_{1} u_{0}(x) w(x) .
\end{gathered}
$$

Hence we deduce from equations (1) and (3) that

$$
\begin{aligned}
k_{0}^{\prime} v_{0} \int_{C} H(x, y)\left[u(y)-u_{0}(y)\right] & d y \\
= & \int_{C} H(x, y) u(y)[v(y)-w(y)] d y-k_{0}^{\prime} v_{0} \\
& =v(x)-w(x)-k_{0}^{\prime}, v_{0}=0,
\end{aligned}
$$

so that equation (2) must hold when the inequality (6) becomes an equality.

Conversely, if $u(x)$ satisfies (2), we multiply both sides by $u_{0}(x)$ and integrate. Using equation (3) and the symmetry of $H$, we deduce that

$$
\int_{C} u(x) d x=\int_{C} u_{0}(x) d x .
$$

This completes the proof of the theorem.

It should be noted that if $H(x, y)$ is closed in the space of bounded measurable functions, then equation (2) can hold only when $u(x)=u_{0}(x)$ 
almost everywhere. This hypothesis is satisfied in all of the physical examples known so far.

In addition it generally happens that $\lambda_{1}<0$, so that the inequality $u(x) \geqq \lambda_{1} u_{0}(x)$ is vacuously true when $u(x)$ is in $U$, i.e., a true absolute minimum occurs.

\section{BIBLIOGRAPHY}

1. G. Goertzel, Minimum critical mass and flat flux, Journal of Reactor Science and Technology vol. 2 (1952) pp. 19-25 (declassified 11/10/52, CF-52-2-9).

2. A. C. Zaanen, On the theory of linear integral equations, III, Neder. Akad Wetensch. Amsterdam vol. 49 (1946) pp. 292-301.

Nuclear Development Associates, Inc.

\section{A CHARACTERIZATION OF ANALYTIC FUNCTIONS ${ }^{1}$}

J. E. MCLAUGHLIN AND C. J. TITUS

1. Let $f$ be a mapping of an open set $D$ in the $x y$-plane into the $u v$-plane where the component functions $u$ and $v$ are continuously differentiable. If the mapping is sense preserving, then the Jacobian, $J(f)$, cannot be negative at any point. If, by analogy with analytic functions, one assumes also that the Jacobian is zero only if the Jacobian matrix has rank zero, then one is led to the study of a family of mappings $\mathfrak{F}$ where

$$
\begin{gathered}
f \in \mathfrak{F} \rightarrow J(f) \geqq 0, \\
J(f)=0 \rightarrow \text { rank of } J \text { is zero. }
\end{gathered}
$$

The purpose of this paper is in part to show that if any real linear vector space of mappings $\mathfrak{B}$ is contained in $\mathfrak{F}$ and $\mathfrak{B}$ contains a pair of analytic functions whose derivatives are independent on $D$, then $\mathfrak{B}$ contains only analytic functions.

We first prove an algebraic lemma upon which the whole characterization rests.

2. Let $\mathbb{S}$ be the vector space of all $2 \times 2$ matrices of the form

$$
\left(\begin{array}{rr}
a & -b \\
b & a
\end{array}\right)
$$

Received by the editors October 28, 1953.

1 The work in this paper was sponsored in part by Office of Naval Research. 